\title{
A Generalization of a Class of Test Matrices
}

\author{
By Robert J. Herbold
}

Abstract. We consider matrices of the following form: $G_{n}\left(a_{1}, a_{2}, \cdots, a_{n-1}, b_{1}, b_{2}, \cdots, b_{n}\right)=$ $\left(\beta_{i, j}\right), 1 \leqq i, j \leqq n$, where $a_{1}, \cdots, a_{n-1}, b_{1}, \cdots, b_{n}$ are constants and

$$
\beta_{i, j}=b_{j}, \quad j \geqq i ; \quad \beta_{i j}=a_{j}, j<i .
$$

We deduce in analytic form the determinant, inverse matrix, characteristic equation, and eigenvectors of $G_{n}$. Knowing these properties enables us to generate valuable test matrices by appropriately selecting the order and elements of $G_{n}$.

1. Introduction. In [1], matrices of the following form were considered:

$$
K_{n} \equiv K_{n}\left(a_{1}, a_{2}, \cdots, a_{n-1}\right)=\left(\alpha_{i, j}\right), \quad 1 \leqq i, j \leqq n,
$$

where $a_{1}, a_{2}, \cdots, a_{n-1}$ were constants and

$$
\alpha_{i, j}=1, \quad j \geqq i ; \quad \alpha_{i j}=a_{j}, \quad j<i .
$$

Therefore,

$$
K_{n}=\left[\begin{array}{lllllcl}
1 & 1 & \cdot & \cdot & \cdot & 1 & 1 \\
a_{1} & 1 & \cdot & \cdot & \cdot & 1 & 1 \\
a_{1} & a_{2} & \cdot & \cdot & \cdot & 1 & 1 \\
\cdot & \cdot & \cdot & \cdot & \cdot & \cdot & \cdot \\
a_{1} & a_{2} & \cdot & \cdot & \cdot & 1 & 1 \\
a_{1} & a_{2} & \cdot & \cdot & \cdot & a_{n-1} & 1
\end{array}\right] .
$$

The determinant, inverse matrix, characteristic equation, and eigenvectors were deduced in analytic form. Consequently, these matrices are valuable test matrices for evaluating the accuracy and efficiency of computational procedures. We shall consider the class of matrices of the following more general form:

$$
G_{n} \equiv G_{n}\left(a_{1}, a_{2}, \cdots, a_{n-1}, b_{1}, b_{2}, \cdots, b_{n}\right)=\left(\beta_{i, j}\right), \quad 1 \leqq i, j \leqq n,
$$

where $a_{1}, \cdots, a_{n-1}, b_{1}, \cdots, b_{n}$ are constants and

$$
\beta_{i, j}=b_{j}, \quad j \geqq i ; \quad \beta_{i j}=a_{j}, \quad j<i .
$$

Thus

$$
G_{n}=\left[\begin{array}{ccccccc}
b_{1} & b_{2} & \cdot & \cdot & \cdot & b_{n-1} & b_{n} \\
a_{1} & b_{2} & \cdot & \cdot & \cdot & b_{n-1} & b_{n} \\
a_{1} & a_{2} & \cdot & \cdot & \cdot & b_{n-1} & b_{n} \\
\cdot & \cdot & \cdot & \cdot & \cdot & \cdot & \cdot \\
a_{1} & a_{2} & \cdot & \cdot & \cdot & b_{n-1} & b_{n} \\
a_{1} & a_{2} & \cdot & \cdot & \cdot & a_{n-1} & b_{n}
\end{array}\right] .
$$

We will assume $b_{n} \neq 0$. Obviously (1) is a special case of (2). We shall deduce the determinant, inverse matrix, characteristic equation and eigenvectors of $G_{n}$ in the same way these properties of $K_{n}$ were derived in [1].

Received February 3, 1969. 
2. The Properties of $G_{n}$. The determinant of $G_{n}$ in (2) is $b_{n} \prod_{j=1}^{n-1}\left(b_{j}-a_{j}\right)$. This can be seen by subtracting $a_{j} / b_{n}$ times the last column of $G_{n}$ from the $j$ th column for $1 \leqq j \leqq n-1$ to obtain

$$
\left[\begin{array}{ccccccc}
\left(b_{1}-a_{1}\right) & \left(b_{2}-a_{2}\right) & . & . & . & \left(b_{n-1}-a_{n-1}\right) & b_{n} \\
0 & \left(b_{2}-a_{2}\right) & . & . & . & \left(b_{n-1}-a_{n-1}\right) & b_{n} \\
0 & 0 & . & . & . & \left(b_{n-1}-a_{n-1}\right) & b_{n} \\
. & . & . & . & . & \cdot & . \\
0 & 0 & . & . & \cdot & \left(b_{n-1}-a_{n-1}\right) & b_{n} \\
0 & 0 & . & . & . & 0 & b_{n}
\end{array}\right] .
$$

This matrix has the same determinant as $G_{n}$, namely $b_{n} \prod_{j=1}^{n-1}\left(b_{j}-a_{j}\right)$.

Since we are assuming $b_{n} \neq 0$, we see that $G_{n}$ is a singular matrix if and only if $b_{j}=a_{j}$ for some $j, 1 \leqq j \leqq n-1$.

By inspection we can verify that for $n \geqq 2$, the inverse of $G_{n}$ in (2) is

$$
G_{n}^{-1}=\left(\delta_{i, j}\right)=\left[\begin{array}{cccccccc}
\frac{1}{c_{1}} & \frac{-1}{c_{1}} & 0 & . & . & . & 0 & 0 \\
0 & \frac{1}{c_{2}} & \frac{-1}{c_{2}} & . & . & . & 0 & 0 \\
0 & 0 & \frac{1}{c_{3}} & . & . & . & 0 & 0 \\
\cdot & . & . & . & . & . & . & . \\
0 & 0 & 0 & . & . & . & 0 & 0 \\
0 & 0 & 0 & . & . & . & \frac{-1}{c_{n-2}} & 0 \\
0 & 0 & 0 & . & . & \cdot & \frac{1}{c_{n-1}} & \frac{-1}{c_{n-1}} \\
\frac{-a_{1}}{c_{1} b_{n}} & \frac{d_{1}}{c_{1} c_{2} b_{n}} & \frac{d_{2}}{c_{2} c_{3} b_{n}} & . & . & \cdot \frac{d_{n-2}}{c_{n-2} c_{n-1} b_{n}} & \frac{b_{n-1}}{c_{n-1} b_{n}}
\end{array}\right] \text {, }
$$

where $c_{i}=\left(b_{i}-a_{i}\right), 1 \leqq i \leqq n-1$ and $d_{i}=a_{i} b_{i+1}-a_{i+1} b_{i}, 1 \leqq i \leqq n-2$; that is

$$
\begin{aligned}
\delta_{i, j} & =\frac{1}{\left(b_{i}-a_{i}\right)}, \quad j=i, i \neq n, \\
& =\frac{-1}{\left(b_{i}-a_{i}\right)}, \quad j=i+1, i \neq n, \\
& =\frac{a_{j-1} b_{j}-a_{j} b_{j-1}}{\left(b_{j}-a_{j}\right)\left(b_{j-1}-a_{j-1}\right) b_{n}}, \quad j \neq 1, n ; i=n, \\
& =\frac{-a_{1}}{\left(b_{1}-a_{1}\right) b_{n}}, \quad j=1, i=n, \\
& =\frac{b_{n-1}}{\left(b_{n-1}-a_{n-1}\right) b_{n}}, \quad j=n, i=n, \\
& =0, \quad j>i+1, i<n-1, \\
& =0, \quad j<i, i \neq n .
\end{aligned}
$$


If $n=1, G_{n}^{-1}=1 / b_{1}$.

Assume $\lambda$ is a nonzero eigenvalue of $G_{n}$. If $\mathbf{x}$ is the corresponding eigenvector in the form $\mathbf{x}=\left(1, x_{2}, x_{3}, \cdots, x_{n}\right)^{T}$, then $G_{n} \mathbf{x}=\lambda \mathbf{x}$, which we can write as

$$
\begin{aligned}
& b_{1}+b_{2} x_{2}+\cdots+b_{n-1} x_{n-1}+b_{n} x_{n}=\lambda, \\
& a_{1}+b_{2} x_{2}+\cdots+b_{n-1} \cdot x_{n-1}+b_{n} \cdot x_{n}=\lambda_{1} x_{2} \text {, } \\
& a_{1}+a_{2} x_{2}+\cdots+b_{n-1} x_{n-1}+b_{n} x_{n}=\lambda_{x} x_{3} \text {, } \\
& a_{1}+a_{2} x_{2}+\cdots+a_{n-1} x_{n-1}+b_{n} x_{n}=\lambda x_{n} .
\end{aligned}
$$

By subtracting the first equation from the second in (3), we obtain

$$
x_{2}=\left(\lambda-b_{1}+a_{1}\right) / \lambda .
$$

By subtracting the second equation from the third in (3), and using (4), we obtain

$$
x_{3}=\left(\lambda-b_{2}+a_{2}\right)\left(\lambda-b_{1}+a_{1}\right) / \lambda^{2} .
$$

Continuing in this fashion, we find that

$$
x_{j}=\frac{\prod_{k=1}^{j-1}\left(\lambda-b_{k}+a_{k}\right)}{\lambda^{j-1}}, \quad 2 \leqq j \leqq n .
$$

This determines the eigenvector for $\lambda \neq 0$. Note that if $\lambda \neq 0$, no matter what the multiplicity of $\lambda$ is, there is only one eigenvector corresponding to it.

In order to obtain the characteristic equation of $G_{n}$, we substitute the values of $x_{j}$ in (5) into the first equation of (3) and multiply both sides of this equation by $\lambda^{n-1}$. This yields

$$
\lambda^{n}-b_{1} \lambda^{n-1}-\sum_{j=1}^{n-1}\left\{b_{j+1} \lambda^{n-j-1} \prod_{k=1}^{j}\left(\lambda-b_{k}+a_{k}\right)\right\}=0,
$$

which is the characteristic equation. In the notation of [1], if we let

$$
S_{k, j}=\sum\left(b_{\alpha_{1}}-a_{\alpha_{1}}\right)\left(b_{\alpha_{2}}-a_{\alpha_{2}}\right) \cdots\left(b_{\alpha_{:}}-a_{\alpha_{:}}\right),
$$

with the sum extending over all possible products of the $j$ terms $\left(b_{i}-a_{i}\right), 1 \leqq i \leqq j$, taken in combinations $k$ at a time, then because

$$
\prod_{k=1}^{j}\left(\lambda-b_{k}+a_{k}\right)=\lambda^{j}-S_{1, j} \lambda^{j-1}+S_{2, j} \lambda^{j-2}-\cdots+(-1)^{j} \widehat{S}_{i_{j}},
$$

the characteristic equation in (6) can be written in the form

$$
\begin{aligned}
\lambda^{n}-\left(\sum_{j=1}^{n} b_{j}\right) \lambda^{n-1} & +\left(S_{1,1}+S_{12}+\cdots+S_{1, n-1}\right) \lambda^{n-2} \\
& -\left(S_{2,2}+S_{2,3}+\cdots+S_{2, n-1}\right) \lambda^{n-3} \\
& +\left(S_{3,3}+S_{3,4}+\cdots+S_{3, n-1}\right) \lambda^{n-4} \\
& \cdots \\
& (-1)^{n-1}\left(S_{n-2, n-2}+S_{n-2, n-1}\right) \lambda+(-1)^{n} S_{n-1, n-1}=0 .
\end{aligned}
$$

If zero is an eigenvalue of $G_{n}$, then $b_{i}=a_{i}$, for at least one $i, 1 \leqq i \leqq n$, and the $i$ th column is a multiple of the $n$th column. As in [1], in calculating the characteristic 
equation and the eigenvectors we can simply ignore the columns (and the corresponding rows) which are multiples of the last column and proceed as before with the smaller matrix. We then multiply the resulting characteristic equation by the proper power of $\lambda$ and add to the eigenvectors a basis set for the null space of $G_{n}$.

3. Test Matrices. The test matrix given in [1] corresponds to $G_{n}$ in (2) with $b_{i}=1,1 \leqq i \leqq n$, and $a_{i}=10^{i}-1 / 10^{i}, 1 \leqq i \leqq n-1$. Another interesting and useful test matrix is obtained by letting $b_{i}=1 / 2 i, 1 \leqq i \leqq n$, and $a_{i}=1 / 2 i+1$, $1 \leqq i \leqq n-1$, in (2). This yields the matrix

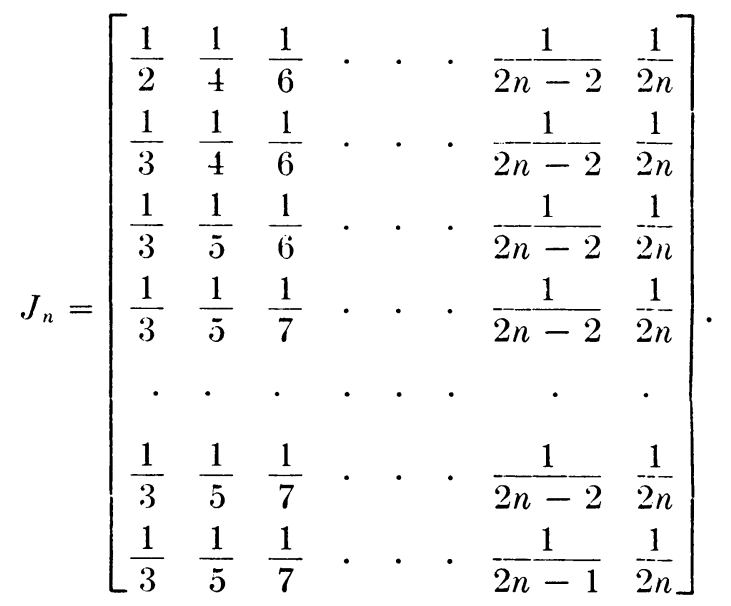

By the results in Section 2, the determinant of $J_{n}$ is $1 /(2 n)$ ! and

$$
\left(J_{n}\right)^{-1}=\left[\begin{array}{rrrrrcc}
6 & -6 & 0 & . & . & 0 & 0 \\
0 & 20 & -20 & . & . & 0 & 0 \\
0 & 0 & 42 & . & . & 0 & 0 \\
. & . & . & . & . & . & 0 \\
0 & 0 & 0 & . & . & 0 & 0 \\
0 & 0 & 0 & . & . & -(2 n-4)(2 n-3) & 0 \\
0 & 0 & 0 & . & . & (2 n-2)(2 n-1) & -(2 n-2)(2 n-1) \\
-4 n & -4 n & -4 n & . & . & -4 n & (2 n)(2 n-1)
\end{array}\right] .
$$

Professional Services Department

Procter and Gamble

Winton Hill Technical Center

Cincinnati, Ohio 45224

1. H. W. Milnes, "A note concerning the properties of a certain class of matrices," Math. Comp., v. 22, 1968, pp. 827-832. 